\title{
Article
}

\section{TOWARD A SOLUTION FOR THE Ca if TRIPLET PUZZLE: RESULTS FROM DWARF ELLIPTICAL GALAXIES}

Dolf, Michielsen, Koleva, Mina, Prugniel, Philippe, Zeilinger, Werner W, De Rijcke, Sven, Dejonghe, Herwig, Pasquali, Anna, Ferreras, Ignacio and Debattista, Victor P

Available at https://clok.uclan.ac.uk/16841/

Dolf, Michielsen, Koleva, Mina, Prugniel, Philippe, Zeilinger, Werner W, De Rijcke, Sven, Dejonghe, Herwig, Pasquali, Anna, Ferreras, Ignacio and Debattista, Victor P orcid iconORCID: 0000-0001-7902-0116 (2007) TOWARD A SOLUTION FOR THE Ca Ii TRIPLET PUZZLE: RESULTS FROM DWARF ELLIPTICAL GALAXIES. The Astrophysical Journal Letters, 670 (2). L101-L104. ISSN 2041-8205

It is advisable to refer to the publisher's version if you intend to cite from the work. http://dx.doi.org/10.1086/524404

For more information about UCLan's research in this area go to http://www.uclan.ac.uk/researchgroups/ and search for < name of research Group>.

For information about Research generally at UCLan please go to http://www.uclan.ac.uk/research/

All outputs in CLoK are protected by Intellectual Property Rights law, including Copyright law. Copyright, IPR and Moral Rights for the works on this site are retained by the individual authors and/or other copyright owners. Terms and conditions for use of this material are defined in the policies page.

\section{CLoK}

Central Lancashire online Knowledge www.clok.uclan.ac.uk

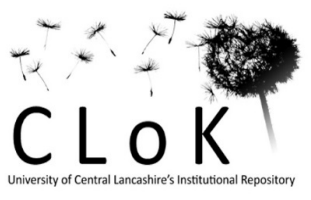


The Astrophysical Journal, 670: L101-L104, 2007 December 1

(C) 2007. The American Astronomical Society. All rights reserved. Printed in U.S.A.

\title{
TOWARD A SOLUTION FOR THE Ca II TRIPLET PUZZLE: RESULTS FROM DWARF ELLIPTICAL GALAXIES
}

\author{
Dolf Michielsen, ${ }^{1}$ Mina Koleva, ${ }^{2,3}$ Philippe Prugniel, ${ }^{2,4}$ Werner W. Zeilinger, ${ }^{5}$ Sven De Rijcke, \\ Herwig Dejonghe, ${ }^{6}$ Anna Pasquali, ${ }^{8}$ Ignacio Ferreras, ${ }^{9}$ and Victor P. Debattista ${ }^{10,11}$ \\ Received 2007 July 16; accepted 2007 October 15; published 2007 October 29
}

\begin{abstract}
We present new estimates of ages and metallicities, based on VLT FORS optical (4400-5500 $\AA$ ) spectroscopy, of 16 dwarf elliptical galaxies (dE's) in the Fornax Cluster and in southern groups. These dE's are more metalrich and younger than previous estimates based on narrowband photometry and low-resolution spectroscopy. For our sample we find a mean metallicity $[\mathrm{Z} / \mathrm{H}]=-0.33$ dex and mean age $3.5 \mathrm{Gyr}$, consistent with similar samples of dE's in other environments (Local Group, Virgo). Three dE's in our sample show emission lines and very young ages. This suggests that some dE's formed stars until a very recent epoch and were self-enriched by a long star formation history. Previous observations of large near-infrared ( $8500 \AA)$ Ca II absorption strengths in these dE's are in good agreement with the new metallicity estimates, solving part of the so-called calcium puzzle.
\end{abstract}

Subject headings: galaxies: dwarf — galaxies: fundamental parameters — galaxies: stellar content

\section{INTRODUCTION}

The observed strength of the near-infrared Ca II triplet absorption lines in early-type galaxies has presented astronomers with an interesting puzzle over the past couple of years. Cenarro et al. (2001) defined a new $\mathrm{CaT}^{*}$ index, carefully correcting for the underlying $\mathrm{H}$ Paschen absorption. Whereas other metallicity tracers, such as $\mathrm{Mg}_{2}$, correlate with velocity dispersion $\sigma$, it was found that $\mathrm{CaT}^{*}$ anticorrelates with $\sigma$ in elliptical galaxies (E's) and in bulges of spiral galaxies (Saglia et al. 2002; Cenarro et al. 2003; Falcón-Barroso et al. 2003). Population synthesis model predictions also show that for subsolar metallicities, $\mathrm{CaT}^{*}$ should be sensitive to metallicity but virtually independent of age, while at supersolar metallicity, the $\mathrm{CaT}^{*}$ saturates (Vazdekis et al. 2003). However, taking metallicities estimated from optical spectra, Saglia et al. (2002) reported that the measured CaT* values in E's are smaller by $0.5 \AA$ than those predicted by population synthesis models. Michielsen et al. (2003, hereafter Paper I) showed that the anticorrelation of $\mathrm{CaT}^{*}$ with $\sigma$ extends into the dwarf elliptical (dE) regime. These dE's were expected to have metallicities of the order of $[\mathrm{Z} / \mathrm{H}] \sim-1$, and ages of the order of $10 \mathrm{Gyr}$ (Held \& Mould 1994; Rakos et al. 2001). The measured CaT*

\footnotetext{
${ }^{1}$ School of Physics and Astronomy, University of Nottingham, University Park, NG7 2RD Nottingham, UK.

${ }^{2}$ Université de Lyon, Lyon, France; Université Lyon 1, Villeurbanne, F69622, France; Centre de Recherche Astronomique de Lyon, Observatoire de Lyon, F-69561, France; CNRS, UMR 5574; Ecole Normale Supérieure de Lyon, Lyon, France.

${ }^{3}$ Department of Astronomy, St. K1. Ohridski University of Sofia, BG-1164 Sofia, Bulgaria.

${ }^{4}$ GEPI Observatoire de Paris-Meudon, 5 place Jules Janssen, Meudon, F92195, France.

${ }^{5}$ Institut für Astronomie, Universität Wien, Türkenschanzstrasse 17, A-1180 Wien, Austria.

${ }^{6}$ Sterrenkundig Observatorium, Ghent University, Krijgslaan 281, S9, B9000 Ghent, Belgium.

${ }^{7}$ Research Postdoctoral Fellow of the Fund for Scientific Research, Flanders, Belgium (FWO).

${ }^{8}$ Max-Planck-Institut für Astronomie, Koenigstuhl 17, D-69117 Heidelberg, Germany.

${ }^{9}$ Physics Department, King's College London, Strand, London WC2R 2LS, UK.

${ }^{10}$ Centre for Astrophysics, University of Central Lancashire, Preston PR1 $2 \mathrm{HE}, \mathrm{UK}$.

${ }^{11}$ RCUK Academic Fellow.
}

values were significantly larger than those expected for such old, metal-poor stellar systems.

All of the proposed solutions to this conundrum, such as variations of the initial mass function or the calcium yield as a function of metallicity or velocity dispersion, require considerable fine-tuning. None of them satisfactorily explains both the small $\mathrm{CaT}^{*}$ in E's and the large $\mathrm{CaT}^{*}$ in $\mathrm{dE}^{\prime}$ 's without creating other difficulties, e.g., with the $\mathrm{FeH} \lambda 9916$ index values observed in bright ellipticals ( $\mathrm{FeH}$ is strong in dwarf stars but nearly absent in giants) and with stellar mass-to-light ratios (Saglia et al. 2002; Cenarro et al. 2003). However, the stellar populations of E's and dE's are most likely not single-age, single-metallicity populations, or SSPs, as was implicitly assumed in essentially all age and metallicity estimates (see, e.g., Pasquali et al. 2005). Moreover, the stars that dominate the blue spectral range (mostly hot dwarf stars) do not necessarily have the same mean ages/metallicities as the stars producing the red light (mostly cool giants). These issues, together with systematic uncertainties inherent to population synthesis tools, potentially contribute to the CaT puzzle.

Because of their low surface brightness, accurate estimates of the ages and metallicities of dE's are still scarce. Recent studies of dE's in the Virgo Cluster (Geha et al. 2003; van Zee et al. 2004) report younger ages and higher metallicities than found in Fornax dE's. In Paper I, the ages and metallicities of the dE's were taken from the literature, where low-resolution, modest-S/N spectroscopic (Held \& Mould 1994) or narrowband photometric (Rakos et al. 2001) techniques were used. As a sanity check, we have now acquired high-resolution, high-S/N optical spectra with VLT FORS of all the dE's for which we presented $\mathrm{CaT}^{*}$ measurements in Paper I. This puts us in a position where we can for the first time compare the CaT* measurements with model predictions based on robust age and metallicity estimates.

\section{OBSERVATIONS AND DATA REDUCTION}

The sample consists of seven dE's in the southern NGC 5044, NGC 5898, and Antlia groups, and nine dE's in the Fornax Cluster. All the observations were carried out in Service Mode at the ESO VLT, in seeing conditions between $0.6^{\prime \prime}$ and $0.9^{\prime \prime}$ FWHM. Integration times varied between 2 and $7 \mathrm{hr}$ per galaxy. The group dE's were observed in 2005 April and May using FORS2 with GRISM_60 OB and a slit width of $0.5^{\prime \prime}$. This 
results in a wavelength range of $3300-6200 \AA$ at a $3.0 \AA$ (FWHM) resolution. The Fornax dE's were observed in 2005 December and 2006 January using FORS1 with the holographic GRISM_1200g and a slit width of 1.0". This results in a wavelength range of 4350-5530 $\AA$ at a $2.6 \AA$ (FWHM) resolution. The data reduction was carried out using the ESO-MIDAS package. ${ }^{12}$ The spectra for each galaxy were bias-subtracted, flat-fielded, corrected for cosmic-ray events, wavelength-calibrated, and co-added. Then the sky was subtracted and the spectra were extinction-corrected and flux-calibrated using spectrophotometric standard stars. For the purpose of this Letter, we extracted 1D spectra over an aperture of radius $R_{e} / 8$ (or $1^{\prime \prime}$ for galaxies with $R_{e}<8^{\prime \prime}$; De Rijcke et al. 2005), to be consistent with the region in which the $\mathrm{CaT}^{*}$ index was measured in Paper I. These spectra have a S/N $>75 \AA^{-1}$ in the wavelength region $4700-5500 \AA$. A full description of the data analysis will be presented in a forthcoming paper (Koleva et al. 2007).

\section{RESULTS}

\subsection{Ages and Metallicities}

To measure the ages and metallicities of the dE's, we compared our observations to single-age, single-metallicity population (SSP) synthesis models. We used two different techniques and models: (1) Thomas et al. (2003, hereafter TMB03) models in the Lick/IDS index system, and (2) Pegase-HR models (Le Borgne et al. 2004) with the ELODIE.3.1 stellar library (Prugniel et al. 2007) to which we perform a full-spectrum fit (Koleva et al. 2007). To transform our spectra to the Lick/IDS system, we smoothed our spectra to the Lick/IDS resolution $(\sim 8.4 \AA \mathrm{FWHM})$ and measured the $\mathrm{H} \beta, \mathrm{Mg} b, \mathrm{Fe} 5270$, and Fe5335 indices (Worthey et al. 1994). In each setup we have three stars in common with the original Lick/IDS library. This number is too small to determine systematic offsets, so we applied the offsets to the Jones library (Worthey \& Ottaviani 1997). These corrected indices agree within the error bars with those given in Worthey \& Ottaviani (1997). We then compared the indices of the dE's with TMB03 models with varying $[\alpha /$ $\mathrm{Fe}]$ abundance ratios, applying a quadratic interpolation over the nine nearest SSP model grid points. The $1 \sigma$ error contours on the derived quantities are calculated by Monte Carlo simulations taking into account the measured errors in the indices (see Cardiel et al. 2003 for a detailed description). Note that systematic errors introduced by the conversion to the Lick/IDS system are not taken into account. This approach yields an estimate for the ages, metallicities, and abundance ratios of the targeted dE's. We find that the dE's have solar abundance ratios and metallicities in the range $-0.7<[\mathrm{Z} / \mathrm{H}]<+0.2$, which justifies the use of the ELODIE.3.1 library to perform the fullspectrum fit. This yields a second, independent, age and metallicity estimate for the program dE's. The $1 \sigma$ errors are computed in a way similar to that with the indices. As is obvious from Figure 1, both techniques agree, with an rms difference of $1.63 \mathrm{Gyr}$ in age and $0.09 \mathrm{dex}$ in $[\mathrm{Z} / \mathrm{H}]$. For a discussion of possible systematic differences between the model predictions, we refer interested readers to Le Borgne et al. (2004). This makes us confident that our age and metallicity estimates are robust. The advantage of the full-spectrum fitting method is that we do not have to convert the data to the (low resolution) Lick/IDS system and that we can carefully take into account

\footnotetext{
${ }^{12}$ The image processing package ESO-MIDAS is developed and maintained by the European Southern Observatory.
}
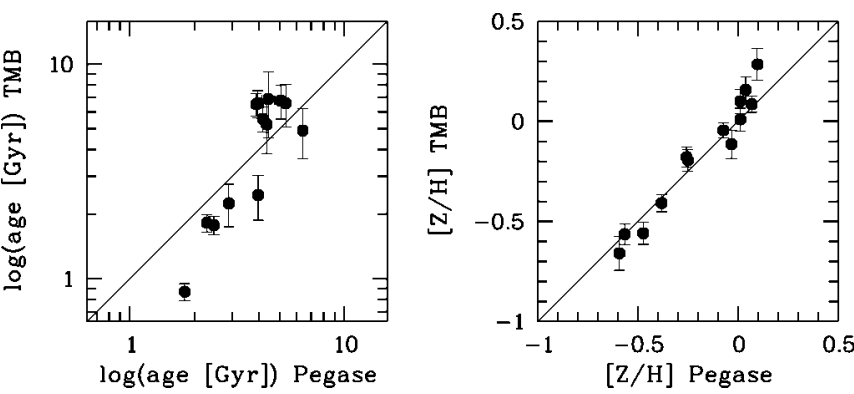

FIG. 1.-Comparison of SSP-equivalent age and metallicity estimates using Lick/IDS indices in combination with TMB03 models and a full-spectrum fit with Pegase-HR models. The error bars are $1 \sigma$ errors. Both approaches clearly agree.

filling by emission lines. Emission is present in three dE's in our sample and we did not study them using the index method. Very low level [O III] (5007 $\AA$ ) emission was detected in FCC 150 , that would not have been detected if the resolution or the $\mathrm{S} / \mathrm{N}$ of the observations were only slightly worse. Columns (2)-(6) of Table 1 give, for our sample of dE's, the ages, metallicities, and $[\alpha / \mathrm{Fe}]$ ratio derived using TMB03, and the age and metallicity derived using Pegase-HR. In the following we use Pegase-HR results. Because the full fit uses all available information in the spectrum, the errors are typically a factor 2-3 smaller than with inversions of bi-index grids.

\subsection{Comparison with Literature Data}

There is clearly a large disparity between the new age and metallicity estimates presented in this Letter and the existing estimates from the literature that needs to be explained. Held \& Mould (1994, hereafter HM94) and Rakos et al. (2001, hereafter R01) studied Fornax dE's and have respectively five and six galaxies in common with our sample. Their metallicity estimates are listed in the last two columns of Table 1. First of all, one of the problems that HM94 faced at the time was the lack of age- and metallicity-dependent population synthesis models. They assumed that the ages of the dE's are comparable to those of Galactic globular clusters in order to justify the use of a metallicity scale calibrated on globular clusters. However, most model bi-index grids are not perpendicular in age and metallicity. At a given measured metal index, a younger age generally implies a higher metallicity. HM94 were aware of this shortcoming at the time and cautioned that their metallicities were probably underestimates. One of the galaxies we have in common with HM94, FCC 207, shows emission lines, indicative of ongoing star formation. We find a very young SSP-equivalent age of 1.5 Gyr. From $\mathrm{H} \alpha$ imaging, we know that the emission in FCC 207 is concentrated in the central 1" (De Rijcke et al. 2003). HM94 did not report emission in FCC 207 (see their Fig. 1), probably because their technique of nodding the telescope perpendicular to the slit to sample the whole nuclear region diluted the emission lines. They did note that the $\mathrm{H} \delta$ absorption of FCC 207 was considerably stronger than the (emission filled) $\mathrm{H} \gamma$ and $\mathrm{H} \beta$ absorption, but attributed this to measurement errors on $\mathrm{H} \delta$.

Second, R01 calibrated their narrowband photometry metallicity scale on the observations of HM94, reobserving all the HM94 dE's. In fact, R01 noted that the metallicity derived from the narrowband colors underestimates the true metallicity, and that the off-set is larger if the population is younger. However they only show 10 and 13 Gyr models, using the latter to compute corrections for their metallicity scale. Using cor- 
TABLE 1

Ages, Metallicities, and $[\alpha / \mathrm{Fe}]$ Ratios

\begin{tabular}{|c|c|c|c|c|c|c|c|}
\hline \multirow[b]{2}{*}{$\begin{array}{c}\text { GaLAXY } \\
\text { NaME } \\
(1)\end{array}$} & \multicolumn{3}{|c|}{ LICK/IDS INDICES: TMB03 } & \multicolumn{2}{|c|}{ Spectrum Fit: Pegase-HR } & \multirow[b]{2}{*}{$\begin{array}{c}\text { HM94 } \\
{\left[\begin{array}{c}\mathrm{Fe} / \mathrm{H}] \\
(\mathrm{dex}) \\
(7)\end{array}\right.}\end{array}$} & \multirow[b]{2}{*}{$\begin{array}{c}\mathrm{R} 01 \\
{[\mathrm{Fe} / \mathrm{H}]} \\
(\mathrm{dex}) \\
(8)\end{array}$} \\
\hline & $\begin{array}{l}\text { Age } \\
\text { (Gyr) } \\
(2)\end{array}$ & $\begin{array}{c}{[\mathrm{Z} / \mathrm{H}]} \\
(\mathrm{dex}) \\
(3)\end{array}$ & $\begin{array}{c}{[\alpha / \mathrm{Fe}]} \\
(\mathrm{dex}) \\
(4)\end{array}$ & $\begin{array}{l}\text { Age } \\
(\mathrm{Gyr}) \\
(5)\end{array}$ & $\begin{array}{c}{[\mathrm{Z} / \mathrm{H}]} \\
(\mathrm{dex}) \\
(6)\end{array}$ & & \\
\hline FCC $043 \ldots \ldots$ & $1.82 \pm 0.17$ & $-0.05 \pm 0.04$ & $0.05 \pm 0.03$ & $2.29 \pm 0.03$ & $-0.07 \pm 0.01$ & $\cdots$ & $\cdots$ \\
\hline $\begin{array}{l}\text { FCC } 046 . \\
\text { FCC } 136\end{array}$ & $\ldots$ & & & $1.15 \pm 0.02$ & $-1.07 \pm 0.02$ & $\ldots$ & $-041 \cdots+012$ \\
\hline FCC 136. & $6.56 \pm 1.48$ & $-0.18 \pm 0.05$ & $0.08 \pm 0.05$ & $5.34 \pm 0.22$ & $-0.26 \pm 0.01$ & & $-0.41 \pm 0.12$ \\
\hline FCC 150 & $6.50 \pm 0.80$ & $-0.41 \pm 0.04$ & $0.00 \pm 0.05$ & $3.88 \pm 0.11$ & $-0.38 \pm 0.01$ & $-0.89 \pm 0.03$ & $-0.75 \pm 0.11$ \\
\hline FCC 204 & $1.77 \pm 0.17$ & $0.09 \pm 0.04$ & $-0.06 \pm 0.03$ & $2.46 \pm 0.04$ & $0.07 \pm 0.01$ & 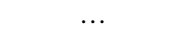 & \\
\hline FCC 207 & & & & $1.47 \pm 0.04$ & $-0.72 \pm 0.03$ & $-1.19 \pm 0.05$ & $-1.51 \pm 0.09$ \\
\hline FCC 245 & $6.75 \pm 1.23$ & $-0.66 \pm 0.09$ & $0.13 \pm 0.08$ & $5.06 \pm 0.32$ & $-0.59 \pm 0.03$ & $-1.00 \pm 0.07$ & $-1.17 \pm 0.10$ \\
\hline FCC 266. & $6.56 \pm 0.97$ & $-0.56 \pm 0.06$ & $-0.01 \pm 0.05$ & $3.95 \pm 0.16$ & $-0.47 \pm 0.01$ & $-0.84 \pm 0.07$ & $-0.83 \pm 0.11$ \\
\hline FCC $288 \ldots \ldots$ & $5.56 \pm 0.76$ & $-0.56 \pm 0.05$ & $-0.04 \pm 0.06$ & $4.17 \pm 0.23$ & $-0.57 \pm 0.02$ & $\ldots$ & $\ldots$ \\
\hline DW $1 \ldots \ldots \ldots$ & $5.23 \pm 1.42$ & $-0.19 \pm 0.06$ & $0.03 \pm 0.06$ & $4.34 \pm 0.18$ & $-0.26 \pm 0.02$ & $\ldots$ & $\ldots$ \\
\hline DW 2 & 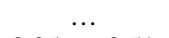 & & & $1.07 \pm 0.02$ & $-0.58 \pm 0.02$ & $\ldots$ & $\ldots$ \\
\hline FS 29 & $2.25 \pm 0.51$ & $0.10 \pm 0.06$ & $-0.09 \pm 0.03$ & $2.89 \pm 0.05$ & $0.01 \pm 0.01$ & $\ldots$ & $\ldots$ \\
\hline FS 75 & $2.45 \pm 0.58$ & $0.16 \pm 0.07$ & $0.02 \pm 0.03$ & $3.96 \pm 0.13$ & $0.04 \pm 0.01$ & $\ldots$ & $\ldots$ \\
\hline FS 76 & $4.91 \pm 1.29$ & $0.29 \pm 0.08$ & $0.03 \pm 0.03$ & $6.40 \pm 0.14$ & $0.09 \pm 0.01$ & $\ldots$ & $\ldots$ \\
\hline FS 131 & $6.88 \pm 2.35$ & $-0.12 \pm 0.07$ & $0.04 \pm 0.06$ & $4.43 \pm 0.31$ & $-0.03 \pm 0.02$ & $\ldots$ & $\ldots$ \\
\hline FS 373. & $0.87 \pm 0.08$ & $0.01 \pm 0.06$ & $0.01 \pm 0.03$ & $1.80 \pm 0.03$ & $0.01 \pm 0.01$ & $\ldots$ & $\ldots$ \\
\hline
\end{tabular}

rections derived for younger ages may reconcile their results with ours.

\subsection{CaT Measurements and Predictions}

Cenarro et al. (2001) defined a set of new indices that quantify the strengths of (1) the Ca II lines (CaT), (2) the Paschen $\mathrm{P} 12$, P14, and P17 lines (PaT), and (3) the Ca II corrected for the absorption contributed by the Paschen P13, P15, and P16 lines $\left(\mathrm{CaT}^{*}=\mathrm{CaT}-0.93 \times \mathrm{PaT}\right)$. In Figure 2 we use our new age and metallicity estimates to compare the observed $\mathrm{CaT}, \mathrm{PaT}$, and $\mathrm{CaT}^{*}$ strengths to the SSP predictions of Vazdekis et al. (2003) with a Salpeter initial mass function (IMF). FS 75 was not observed in the Ca II. For most of the dE's, the values are now consistent with the model predictions. FCC 046

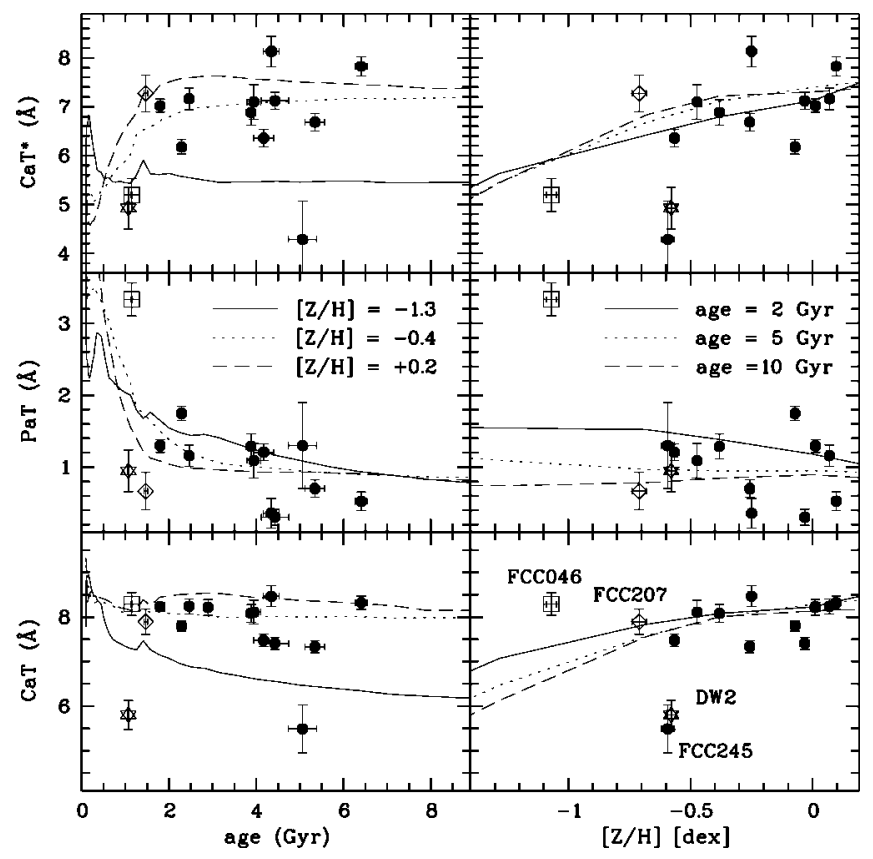

FIG. 2.-CaT, PaT, and CaT* vs. age and metallicity for the Fornax Cluster and southern group dE's. Overlaid are Vazdekis et al. (2003) SSP models with Salpeter a IMF. FCC 046 (square), FCC 207 (diamond), and DW 2 (star) are the three dE's with emission lines. FCC 245 is the most significant outlier. has a high CaT for its age and metallicity. However, this galaxy has emission lines and shows evidence for recent star formation (De Rijcke et al. 2003). This is reflected in the high PaT absorption strength, and the corrected $\mathrm{CaT}^{*}$ agrees very well with the model prediction based on FCC 046's metallicity. The values of the other Fornax dE with emission, FCC 207, agree perfectly well with the model predictions. Surprisingly, in DW 2 we also found emission lines. However, the CaT of DW 2 is already too low compared to the models, and a high PaT would aggravate the situation for CaT*. Finally, FCC 245 appears to be quite normal, morphologically and photometrically speaking, but its $\mathrm{CaT}$ is also lower than expected for its age and metallicity. Still, 13 out of 15 of the observed dE's now have measured ages, metallicities, and $\mathrm{CaT}^{*}$ values that agree with model predictions. The fact that $\mathrm{CaT}^{*}$ values predicted using age/metallicity estimates that were derived from spectral features in the blue part of the spectrum ( 4700-5500 $)$ agree quite reasonably with the observed $\mathrm{CaT}^{*}$ values in the NIR $(\sim 8500 \AA)$ indicates that the SSP assumption is not the cause of the CaT puzzle for dE's. Moreover, the blue Ca $4227 \AA$ line is included in the full-spectrum fit and is generally well reproduced. In the case of the galaxy with the worst fit to this line, FS 076 the residuals do not exceed 5\%.

\section{DISCUSSION AND CONCLUSIONS}

At least in the $\mathrm{dE}$ regime, the $\mathrm{Ca}$ II triplet puzzle seems to be solved. With the new age and metallicity estimates presented in this Letter, the predicted and observed $\mathrm{CaT}^{*}$ indices are in good agreement for all sample galaxies but two. The fact that $\mathrm{CaT}^{*}$ values predicted using age/metallicity estimates that were derived from spectral features in the blue part of the spectrum agree with the observed $\mathrm{CaT}^{*}$ values in the NIR indicates that the SSP assumption is not the cause of the CaT puzzle for $\mathrm{dE}$ 's. Rather, the CaT puzzle in the $\mathrm{dE}$ regime was caused by the spuriously low metallicities and high ages, derived from lower resolution spectra using less sophisticated theoretical models, assigned to Fornax dE's. This shows that, as far as dE's are concerned, the CaT* index, and, in old stellar systems for which the PaT index is small, the CaT index as well, is indeed a good tracer of metallicity. For massive E's, there is still no satisfactory solution for the Ca puzzle. 
This also solves the apparent dichotomy between dE's on the one hand and globular clusters, Local Group dwarf spheroidals (dSph's), and ultracompact dwarfs (UCDs) on the other hand. CaT line strengths measured in individual stars of Local Group dSph's have been shown to be a very accurate tracer of metallicity (Battaglia et al. 2006; Tolstoy et al. 2001) and $[\mathrm{Fe} / \mathrm{H}]$ values derived from $\mathrm{CaT}$ measurements have been used extensively to construct metallicity distributions of the stars in dSph's. Also, for UCDs (Evstigneeva et al. 2007) and globular clusters (Saglia et al. 2002), the CaT index has proved to be an excellent tracer of metallicity. Here, we have shown that in $\mathrm{dE}$ 's as well, the $\mathrm{CaT}^{*}$ index measured from integrated-light spectra can be used as a tracer of metallicity.

To summarize, we derive new age and metallicity estimates for $16 \mathrm{dE}$ 's in the Fornax Cluster and in southern groups using high-S/N optical VLT FORS1+2 spectra. We have measured the $\mathrm{H} \beta, \mathrm{Mg} b, \mathrm{Fe} 5270$, and Fe5335 indices in the Lick/IDS system and applied the TMB03 models to them. We find that these dE's have solar $[\alpha / \mathrm{Fe}]$ abundance ratios. A full-spectrum fit using Pegase-HR with the ELODIE.3.1 stellar library provides us with a second, independent age and metallicity estimate for these galaxies. We find both approaches to be in excellent agreement. With mean metallicity $[\mathrm{Z} / \mathrm{H}]=-0.35$ dex and ages younger than $\approx 7 \mathrm{Gyr}$, these $\mathrm{dE}$ 's are more metalrich and younger than previously thought. Some even show strong emission lines, an indication of ongoing star formation, in agreement with previous $\mathrm{H} \alpha$ imaging of dE's (De Rijcke et al. 2003; Michielsen et al. 2004). The ages and metallicities we derive for the dE's in the Fornax Cluster and in southern groups fall in roughly the same range as those derived by Geha et al. (2003) and van Zee et al. (2004) for dE's in the Virgo Cluster. This is at variance with previous estimates for Fornax dE's which yielded lower metallicities and higher ages (Held \& Mould 1994; Rakos et al. 2001), based on lower resolution spectra and less sophisticated theoretical models. The new age and metallicity estimates are in good agreement with the observed Ca II triplet absorption strengths, solving the calcium puzzle for low-mass systems.

Based on observations collected at the European Southern Observatory, Paranal, Chile (programs 075.B-0179 and 076.B0196). D. M. acknowledges the EU MAGPOP RTN for financial support.

\section{REFERENCES}

Battaglia, G., et al. 2006, A\&A, 459, 423

Cardiel, N., Gorgas, J., Sánchez-Blázquez, P., Cenarro, A. J., Pedraz, S., Bruzual, G., \& Klement, J. 2003, A\&A, 409, 511

Cenarro, A. J., Cardiel, N., Gorgas, J., Peletier, R. F., Vazdekis, A., \& Prada, F. 2001, MNRAS, 326, 959

Cenarro, A. J., Gorgas, J., Vazdekis, A., Cardiel, N., \& Peletier, R. F. 2003, MNRAS, 339, L12

De Rijcke, S., Michielsen, D., Dejonghe, H., Zeilinger, W. W., \& Hau, G. K. T. 2005, A\&A, 438, 491

De Rijcke, S., Zeilinger, W. W., Dejonghe, H., \& Hau, G. K. T. 2003, MNRAS, 339,225

Evstigneeva, E. A., Gregg, M. D., Drinkwater, M. J., \& Hilker, M. 2007, AJ, 133,1722

Falcón-Barroso, J., Peletier, R. F., Vazdekis, A., \& Balcells, M. 2003, ApJ, 588, L17

Geha, M., Guhathakurta, P., \& van der Marel, R. P. 2003, AJ, 126, 1794

Held, E. V., \& Mould, J. R. 1994, AJ, 107, 1307 (HM94)

Koleva, M., Prugniel, P., Ocvirk, P., \& Le Borgne, D. 2007, in IAU Symp. 241, Stellar Populations as Building Blocks of Galaxies, ed. A. Vazdekis $\&$ R. Peletier (Cambridge: Cambridge Univ. Press), 185

Koleva, M., et al. 2007, MNRAS, submitted

Le Borgne, D., Rocca-Volmerange, B., Prugniel, P., Lançon, A., Fioc, M., \& Soubiran, C. 2004, A\&A, 425, 881
Michielsen, D., De Rijcke, S., Dejonghe, H., Zeilinger, W. W., \& Hau, G. K. T. 2003, ApJ, 597, L21 (Paper I)

Michielsen, D., De Rijcke, S., Zeilinger, W. W., Prugniel, P., Dejonghe, H., \& Roberts, S. 2004, MNRAS, 353, 1293

Pasquali, A., Larsen, S., Ferreras, I., Gnedin, O. Y., Malhotra, S., Rhoads, J. E., Pirzkal, N., \& Walsh, J. R. 2005, AJ, 129, 148

Prugniel, P., Soubiran, C., Koleva, M., \& Le Borgne, D. 2007, preprint (astro$\mathrm{ph} / 0703658)$

Rakos, K., Schombert, J., Maitzen, H. M., Prugovecki, S., \& Odell, A. 2001, AJ, 121, 1974 (R01)

Saglia, R. P., Maraston, C., Thomas, D., Bender, R., \& Colless, M. 2002, ApJ, 579, L13

Thomas, D., Maraston, C., \& Bender, R. 2003, MNRAS, 339, 897 (TMB03)

Tolstoy, E. Irwin, M. J., Cole, A. A., Pasquini, L., Gilmozzi, R., \& Gallagher, J. S. 2001, MNRAS, 327, 918

van Zee, L., Barton, E. J., \& Skillman, E. D. 2004, AJ, 128, 2797

Vazdekis, A., Cenarro, A. J., Gorgas, J., Cardiel, N., \& Peletier, R. F. 2003, MNRAS, 340, 1317

Worthey, G., Faber, S. M., Gonzalez, J. J., \& Burstein, D. 1994, ApJS, 94, 687

Worthey, G., \& Ottaviani, D. L. 1997, ApJS, 111, 377 